\title{
Supporting Information for \\ Aerosol-induced changes in the vertical structure of precipitation: a perspective of TRMM precipitation radar
}

Jianping Guo ${ }^{1}$, Huan Liu ${ }^{1}$, Zhanqing Li²*, Daniel Rosenfeld ${ }^{3}$, Mengjiao Jiang ${ }^{4}$, Weixin $\mathrm{Xu}^{4}$, Jonathan H. Jiang ${ }^{5}$, Jing $\mathrm{He}^{1}$, Dandan Chen ${ }^{1}$, Min Min ${ }^{6}$, and Panmao Zhai ${ }^{1}$

\footnotetext{
${ }^{1}$ State Key Laboratory of Severe Weather, Chinese Academy of Meteorological Sciences, Beijing 100081, China

${ }^{2}$ Department of Atmospheric and Oceanic Sciences \& Earth System Science Interdisciplinary Center, University of Maryland, College Park, Maryland 20740, USA

${ }^{3}$ The Hebrew University of Jerusalem, Jerusalem 91904, Israel

${ }^{4}$ College of Atmosphere Science, Chengdu University of Information Technology, Chengdu 610225, China

${ }^{5}$ Department of Atmospheric Sciences, Colorado State University, Fort Collins, Colorado 80523, USA

${ }^{6}$ Jet Propulsion Laboratory, California Institute of Technology, Pasadena, California 91109, USA

${ }^{7}$ NationalSatelliteMeteorological Center, China Meteorological Administration, Beijing 100081, China
}

Correspondence to: Dr. Zhanqing Li (zli@atmos.umd.edu) 


\section{Correlation between environmental parameters and $P M_{10}$}

To see whether clouds have any effects on aerosols, correlation analyses between cloud cover and ground-based $\mathrm{PM}_{10}$ have been performed over the PRD region. As shown in Figure S1, the correlation between cloud cover from MODIS/Aqua and ground-based $\mathrm{PM}_{10}$ over the PRD region is negligible. It could be partly due to the fact that $\mathrm{PM}_{10}$ concentration is measured on the ground, which is not affected by the sky conditions at all.

Also, we performed correlation analyses between environmental parameters such as pressure vertical velocity, wind shear, low troposphere stability (LTS), and moisture flux divergence (A), and $\mathrm{PM}_{10}$ concentrations. The results are shown in Figure S2. No significant correlations are seen, partly because $\mathrm{PM}_{10}$ concentrations are a function of emissions over the PRD region.

\section{Z-R relationship stratified by $P M_{10}$}

Both rain rate and radar reflectivity for three categories of rainfall have been derived, so it is possible to estimate how the Z-R relationship might vary with aerosol content. Figure S3 describes how the Z-R relationship varies with aerosol content. It is difficult to tell whether the Z-R relationship changes as aerosol loading changes. This is likely due to the fact that the Z-R relationship is largely affected by hydrometeor size, meteorological factors, among others, rather than aerosol particles. In addition, the radar reflectivity caused by aerosol particles can be negligible compared with hydrometeors. 


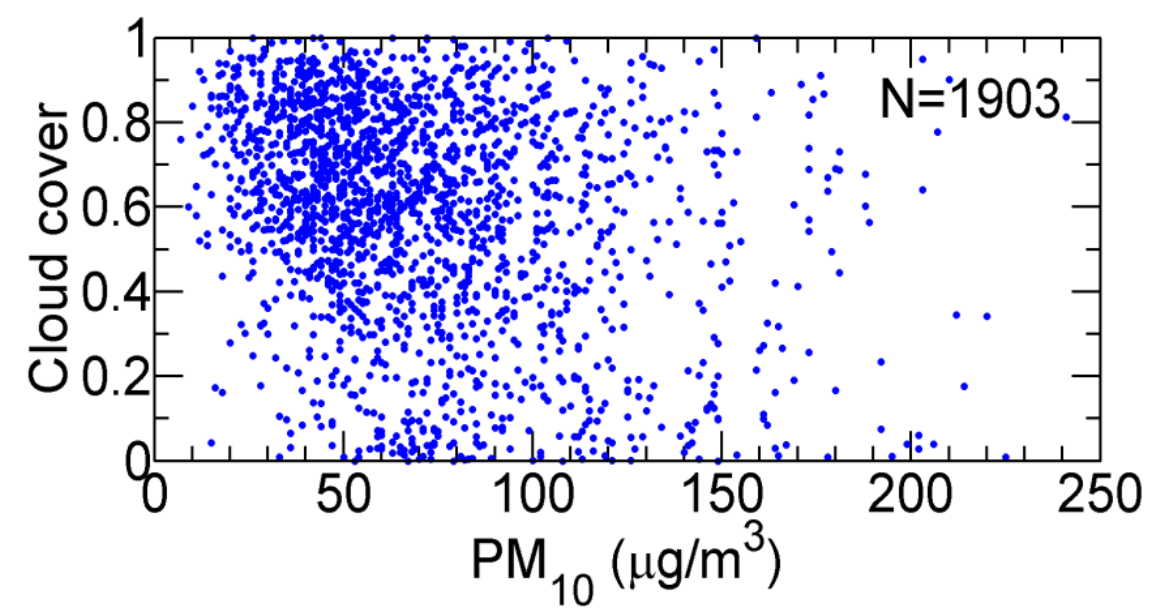

Figure S1. Scatter plot showing the relationship between cloud cover from MODIS/Aqua and ground-based measured PM10 over the PRD region. The red line represents the least-squares regression line. The sample size is given by $N$.
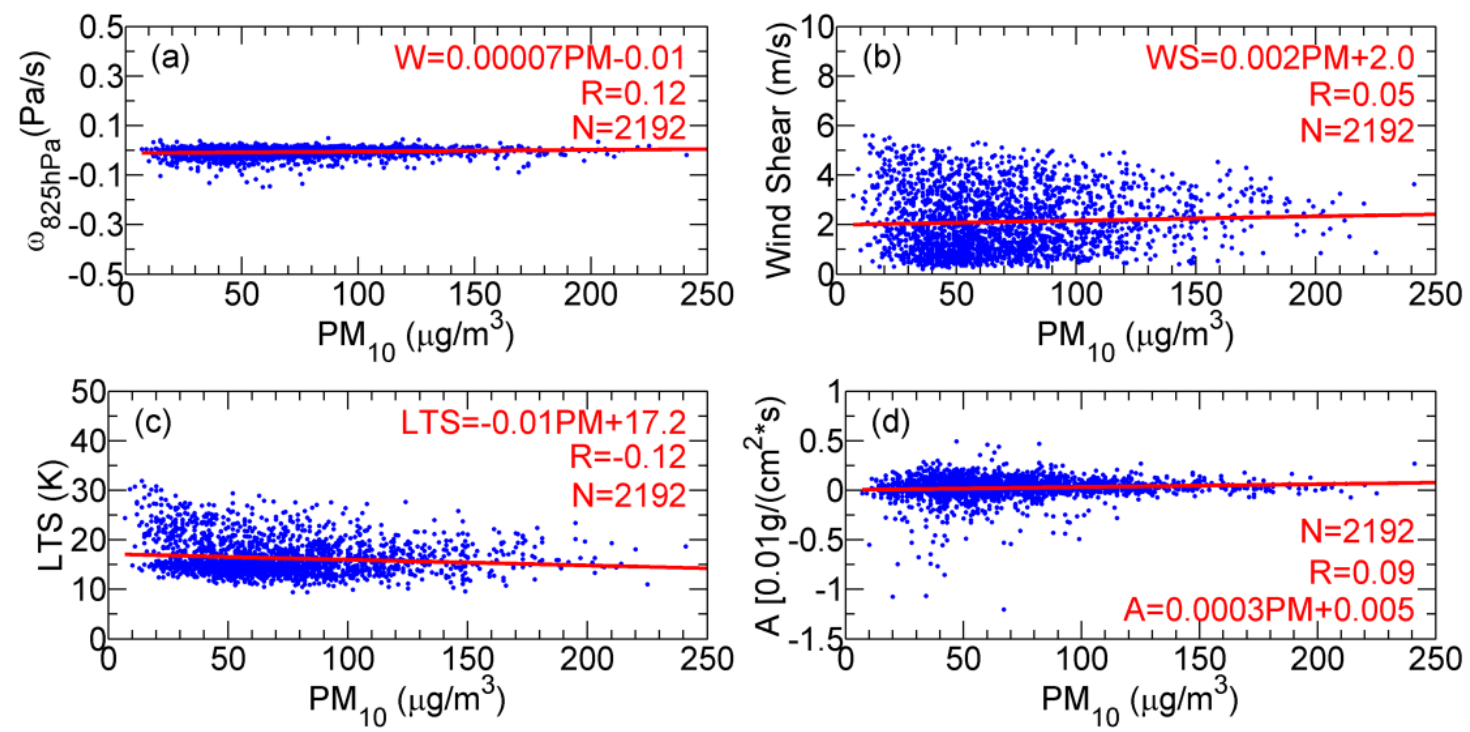

Figure S2. Scatter plots showing the relationships between (a) pressure vertical velocity, (b) vertical wind shear, (c) LTS, and (d) A as a function of ground-based measured $\mathrm{PM}_{10}$ over the PRD region. The red lines represent least-squares regression lines. 

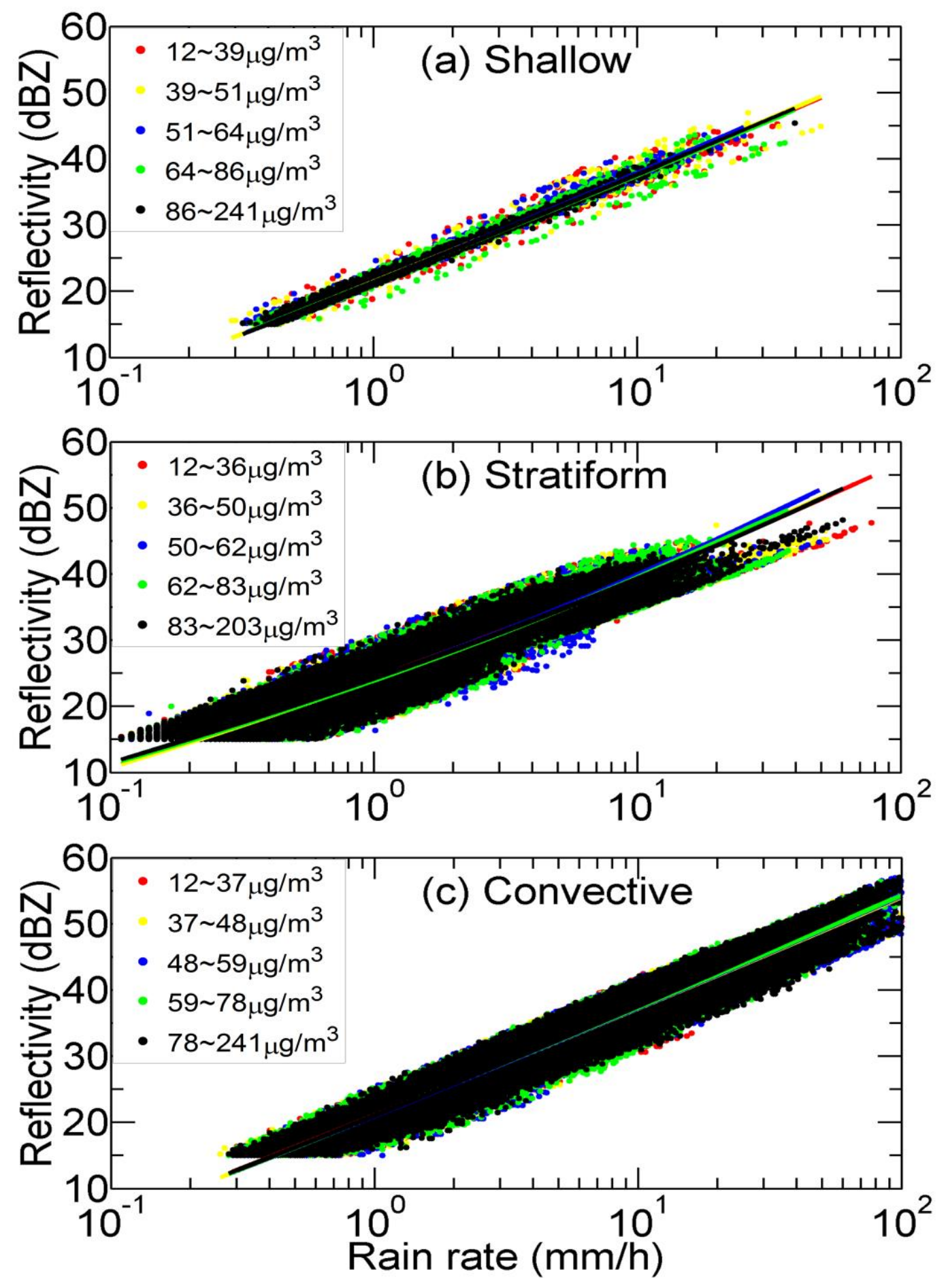

Figure S3. Radar reflectivity as a function of rain rate in different $\mathrm{PM}_{10}$ concentration bins for shallow, stratiform, and convective rain types. 
Table S1. Criteria used to classify the precipitation intensity type and statistics describing each precipitation intensity type in general, and under clean and polluted conditions. The percentage numbers denote the ratio of number of profiles for light, moderate and heavy convective precipitation subtype, respectively, to the total radar profiles. Data are from TRMM PR retrievals made over the PRD region.

\begin{tabular}{cccc}
\hline $\begin{array}{c}\text { Precipitation } \\
\text { intensity }\end{array}$ & Filtering criteria & Clean (\%) & Polluted (\%) \\
\hline Light & $\leqslant 10 \mathrm{~mm} / \mathrm{h}$ & 36.4 & 34.8 \\
Moderate & $10-20 \mathrm{~mm} / \mathrm{h}$ & 33.1 & 40.0 \\
Heavy & $\geqslant 20 \mathrm{~mm} / \mathrm{h}$ & 21.0 & 46.8 \\
\hline
\end{tabular}

